\title{
CONSTRUCTION OF WAVEFORM LIBRARY IN COGNITIVE RADAR
}

\author{
Guo Yongqiang, Ph.D D., \\ Beijing Institute of Tracking and Telecommunications Technology, Beijing, China \\ Wu yumin, MS., \\ Liu Hui, Ph.D D., \\ Communication Engineering, Xiamen University, Xiamen, Fujian, China
}

\begin{abstract}
Based on the thoughts of cognitive radar, Fractional Fourier Transform (FrFT) is used to generate a rotatable waveform libraries of Frank coded/Barker coded waveform in this paper. Then, the ambiguity function is used to analyze the delay resolution, Doppler resolution, delay side-lobe level, and Doppler side-lobe level of the waveform libraries and orthogonality of them is also analyzed. Furthermore, we proved theoretically that there is a fixed coordinate transformation between the waveforms of library and its origin waveform. Therefore, the Cramér-Rao low bound (CRLB) of motion parameters can be computed easily using the waveforms of the libraries, which facilitate the subsequent waveform scheduled work. Simulation results show that the library waveforms can reduce delay resolution to satisfy the different situations and can bring significant benefits for delay resolution, orthogonality and reuse interval.
\end{abstract}

Keywords: Fractional Fourier Transform; Ambiguity Function; Barker; Frank; Waveform Libraries

\section{INTRODUCTION}

The cognitive radar is a new radar system for target detection, tracking, imaging and recognition [1]. Different from the fix transmitted waveform used in the traditional radar, it can schedule the different transmitted waveform adaptively according to the prior information provided by a feedback system interacting with the surrounding environment [2]. And the key parts of it are optimization and selection of the transmitted waveforms. Waveform optimizing and selecting adaptively has been used for such organism as bats and dolphins for hundreds of years [3]. However, the limitation of hardware devices in the transmission terminal such as the ADC limits the technologies of waveform optimization and selection [4]. With the development of software radio technology, software-drive waveform generator has been developed in recent years and [5]. Therefore, more and more researchers have paid attention to the waveform optimization and selection. Although in the practical radar system or even in the experiment platform, the software-drive waveform generators have not been used yet, a number of research and simulation results show that the performance of target detection and tracking has been greatly improved in varieties of occasions by using it [6]. Lack of real-time knowledge about the information state and specificity of the performance goal is mitigated by creating a library sufficiently rich that it is highly likely to contain a waveform providing good performance for every real-time performance criterion and every information state that will be encountered in actual real-time operation. The goal of offline waveform library design is thus to produce a waveform library that is parsimonious, yet sufficient to provide a choice of highperformance waveform against operational measure of effectiveness that vary across a range of possible environments and unknown target states.

Nowadays, Xia Hongen [6] presented that by constructing the linear frequency modulation (LFM) can improve the target tracking performance greatly in radar. Yu Daobin [7] constructed a waveform library including the triangular impulse signal, modulation Gaussian pulse signal and 
the gauss linear modulation pulse signal. He verified that the algorithm combined waveform scheduling indeed improve the performance of target tracking. Reference 8 used Fractional Fourier Transform (FrFT) to modulate an OFDM signal. This technique reduced the interference between the subcarrier, improved SNR and tolerated the bit error rate (BER). Jinbiao [9] designed the rotating waveform library based on rectangular pulse waveform using FrFT and analyzed the relationship between the rotating waveform library and the rectangular pulse waveform. C.Clemente [10] designed the rotating waveform library of phase coding signals, and analyzed the waveform performances such as the delay resolution and the side lobe level and so on.

Inspired by reference 10 and 11, the paper constructs a rotating waveform library based on Frank coded and Barker coded signal using FrFT. And the performance of the generated waveforms in waveform library is analyzed in detail using the ambiguity function. Research and simulation shows that the rotating waveform library using FrFT can be easily and flexibly implemented. And there is a linear transformation between the waveforms in rotating waveform library and the traditional waveform, which is $\left[\begin{array}{l}u \\ v\end{array}\right]=\left[\begin{array}{cc}\cos \alpha & \sin \alpha \\ -\sin \alpha & \cos \alpha\end{array}\right]\left[\begin{array}{c}\tau \\ 2 \pi f_{d}\end{array}\right]$.

Thus, the Cramér-Rao bounds (CRLBs) of motion parameter estimation for the waveforms in rotating waveform library will be calculated easily. And it is convenient to the waveform scheduling. At the same time, the simulation results show that the delay resolution of the waveforms in rotating waveform library can meet the demand of different scenarios. And they have the good features of orthogonality and multiplexing. The rotating waveform library can be applied in the fields such as the frequency reuse Doppler tracking system, the low probability of intercept radar and multiple input multiple output (MIMO) system.

\section{FRACTIONAL FOURIER TRANSFORM (FRFT)}

FrFT was applied in signal processing as a kind of timefrequency representation $[11,12,13]$. FrFT of $x(t)$ is defined as follow:

$$
X_{\alpha}(u)=\left\{F_{\alpha}[x(t)]\right\}(u)=\int_{-\infty}^{+\infty} K_{\alpha}(u, t) x(t) d t
$$

Where $\alpha$ is the rotation angle and $K_{\alpha}(u, t)$, named FrFT kernel, is defined as follows:

$$
K_{\alpha}(u, t)=\left\{\begin{array}{c}
\sqrt{\frac{1-j \cot \alpha}{2 \pi}} e^{\left(j \frac{t^{2}+u^{2}}{2} \cot \alpha-j u t \csc \alpha\right)}, \alpha \neq n \pi \\
\delta(t-u), \alpha=2 n \pi \\
\delta(t+u), \alpha=(2 n \pm 1) \pi
\end{array}\right.
$$

Thus:

$$
\begin{aligned}
X_{\alpha}(u)= & \left\{F_{\alpha}[x(t)]\right\}=\int_{-\infty}^{+\infty} K_{\alpha}(u, t) x(t) d t \\
= & \left\{\begin{array}{c}
\sqrt{\frac{1-j \cot \alpha}{2 \pi}} \int_{-\infty}^{+\infty} e^{\left(j \frac{t^{2}+u^{2}}{2} \cot \alpha-j u t \csc \alpha\right)} x(t) d t, \alpha \neq n \pi \\
x(t), \alpha=2 n \pi \\
x(-t), \alpha=(2 n+1) \pi
\end{array}\right.
\end{aligned}
$$

Where, $\alpha=p \times \frac{\pi}{2}$, when $p=1$ equation(3) is just Fourier transform, while $p=-1$, it is just inverse Fourier transform, where $p$ is the fractional transformation order.

\section{CONSTRUCT WAVEFORM LIBRARIES BASED ON FRFT}

First a basic signal $\Omega$ (e.g. $\Omega=13$ for Barker 13 code ) and the sampling rate $r$ are defined to construct waveform library using FrFT. Therefore, the total length of the digital signal is $N=\Omega \times r$.Using Barker code as an example, the original signals can be written as:

$$
s(n)=\sum_{k=1}^{N} c_{k} \delta(n-k)
$$

Where $c=\left[c_{1}, c_{2}, c_{3}, \ldots, c_{N}\right]$ is supposed to be $\mathrm{N}$ samples of the original signals.

Then, the FrFT is applied to (4):

$$
\begin{aligned}
S_{\alpha}(u) & =F \mathrm{r} F T_{\alpha}[s(n)]=F \mathrm{r} F T_{\alpha}\left[\sum_{k=1}^{N} c_{k} \delta(n-k)\right] \\
& =\sum_{k=1}^{N} c_{k} F r F T_{\alpha}[\delta(n-k)]
\end{aligned}
$$

Due to:

$$
\begin{aligned}
& \operatorname{FrFT}_{\alpha}[\delta(t-\tau)]=\int_{-\infty}^{+\infty} K_{\alpha}(u, t) \delta(t-\tau) d t \\
& =\sqrt{\frac{1-j \cot \alpha}{2 \pi}} \int_{-\infty}^{+\infty} e^{\left(j \frac{t^{2}+u^{2}}{2} \cot \alpha-j u t \csc \alpha\right)} \delta(t-\tau) d t, \alpha \neq n \pi \\
& =\sqrt{\frac{1-j \cot \alpha}{2 \pi}} e^{j \frac{\tau^{2}+u^{2}}{2} \cot \alpha-j u \tau \csc \alpha}, \alpha \neq n \pi
\end{aligned}
$$

Introducing the formula (6) into the formula (5), we get:

$$
\begin{aligned}
& S_{\alpha}(u)=F \mathrm{r} F T_{\alpha}[s(n)] \\
& =\sqrt{\frac{1-j \cot \alpha}{2 \pi}} \sum_{k=1}^{N} c_{k} e^{j \frac{k^{2}+u^{2}}{2} \cot \alpha-j u k \csc \alpha}
\end{aligned}
$$


From formula (7), the rotating waveform can be seen as a group of weighted waveforms of traditional waveform.

Different fractional order $p$ is correspond to a different angle $\alpha$. And different angle $\alpha$ is correspond to different waveform. Therefore, by replacing $u$ with $t$ in formula (7), the waveforms $S=\left[S_{\alpha_{1}}(t), S_{\alpha_{2}}(t), \ldots, S_{\alpha}(t), \ldots, S_{\alpha_{L}}(t)\right]$ constitute the rotating waveform library. Where, $p \in[0,2]$ and $L$ represents the number of waveforms in the library.

\section{CONSTRUCT WAVEFORM LIBRARIES BASED ON FRFT}

Ambiguity function of waveform in libraries is defined as:

$$
\chi_{\alpha}\left(\tau, f_{d}\right)=\int_{-\infty}^{\infty} S_{\alpha}(t) S_{\alpha}^{*}(t-\tau) e^{-j 2 \pi f_{d} t} d t
$$

Supposed the traditional signal is $x(t)$, its ambiguity function can be written as:

$$
\chi_{0}\left(\tau, f_{d}\right)=\int_{-\infty}^{\infty} x(t) x^{*}(t-\tau) e^{-j 2 \pi f_{d} t} d t
$$

According to the translation and symmetry propertiesof FrFT kernel function $[12,14]$ :

$x^{*}(t-\tau)=\int_{-\infty}^{+\infty} S_{\alpha}^{*}(z-\tau \cos \alpha) e^{-j \frac{\tau^{2}}{2} \sin \alpha \cos \alpha+j z \tau \sin \alpha} K_{\alpha}(z, \tau) d z$

Introducing formula (10) into formula (9), we get:

$$
\begin{aligned}
& \chi_{0}\left(\tau, f_{d}\right)=\int_{-\infty}^{\infty} x(t) x^{*}(t-\tau) e^{-j 2 \pi f_{d} t} d t \\
& =\int_{-\infty}^{+\infty} x(t) \int_{-\infty}^{+\infty} S_{\alpha}^{*}(z-\tau \cos \alpha) e^{-j \frac{\tau^{2}}{2} \sin \alpha \cos \alpha+j z \tau \sin \alpha} K_{\alpha}(\tau, z) d z e^{-j 2 \pi f_{d} t} d t \\
& =\int_{-\infty}^{+\infty} \int_{-\infty}^{+\infty} x(t) S_{\alpha}^{*}(z-\tau \cos \alpha) e^{-j \frac{\tau^{2}}{2} \sin \alpha \cos \alpha+j z \tau \sin \alpha} K_{\alpha}(\tau, z) e^{-j 2 \pi f_{d} t} d t d z \\
& =\int_{-\infty}^{+\infty} S_{\alpha}^{*}(z-\tau \cos \alpha) e^{-j \frac{\tau^{2}}{2} \sin \alpha \cos \alpha+j z \tau \sin \alpha} \int_{-\infty}^{+\infty} x(t) K_{\alpha}(\tau, z) e^{-j 2 \pi f_{d} t} d t d z
\end{aligned}
$$

According to the exponent properties of FrFT [15]:

$$
\begin{aligned}
\chi_{0}\left(\tau, f_{d}\right) & =\int_{-\infty}^{+\infty} S_{\alpha}^{*}(z-\tau \cos \alpha) e^{-j \frac{\tau^{2}}{2} \sin \alpha \cos \alpha+j z \tau \sin \alpha} \\
& \cdot \int_{-\infty}^{+\infty} x(t) K_{\alpha}(\tau, z) e^{-j 2 \pi f_{d} t} d t d z \\
=\int_{-\infty}^{+\infty} S_{\alpha}^{*}(z-\tau \cos \alpha) e^{-j \frac{\tau^{2}}{2} \sin \alpha \cos \alpha+j z \tau \sin \alpha} & \cdot S\left(z+2 \pi f_{d} \sin \alpha\right) e^{-j \frac{\left(-2 \pi f_{d}{ }^{2}\right.}{2} \sin \alpha \cos \alpha+j z\left(-2 \pi f_{d}\right) \cos \alpha} d z
\end{aligned}
$$

$$
\begin{aligned}
& \text { If, } \quad \varepsilon=z+2 \pi f_{d} \sin \alpha \\
& \Delta=e^{-j \frac{\tau^{2}}{2} \sin \alpha \cos \alpha-j\left(2 \pi f_{d} \sin \alpha\right) \tau \sin \alpha} e^{-j \frac{\left(-2 \pi f_{d}{ }^{2}\right.}{2} \sin \alpha \cos \alpha+j\left(-2 \pi f_{d} \sin \alpha\right)\left(-2 \pi f_{d}\right) \cos \alpha}
\end{aligned}
$$

formula (12) can be rewritten as:

$\chi_{0}\left(\tau, f_{d}\right)=\int_{-\infty}^{+\infty} S_{\alpha}(\varepsilon) S_{\alpha}^{*}\left(\varepsilon-2 \pi f_{d} \sin \alpha-\tau \cos \alpha\right) e^{-j \varepsilon\left(-\tau \sin \alpha+2 \pi f_{d} \cos \alpha\right)} \Delta d \varepsilon$

$=\Delta \cdot \int_{-\infty}^{+\infty} S_{\alpha}(\varepsilon) S_{\alpha}^{*}\left(\varepsilon-\left(\tau \cos \alpha+2 \pi f_{d} \sin \alpha\right)\right) e^{-j \varepsilon\left(-\tau \sin \alpha+2 \pi f_{d} \cos \alpha\right)} d \varepsilon$

Supposed $\left[\begin{array}{l}u \\ v\end{array}\right]=\left[\begin{array}{cc}\cos \alpha & \sin \alpha \\ -\sin \alpha & \cos \alpha\end{array}\right]\left[\begin{array}{c}\tau \\ 2 \pi f_{d}\end{array}\right]$, formula (13) can be rewritten as:

$$
\chi_{0}\left(\tau, f_{d}\right)=\Delta \cdot \int_{-\infty}^{+\infty} S_{\alpha}(\varepsilon) S_{\alpha}^{*}(\varepsilon-u) e^{-j \varepsilon v} d \varepsilon
$$

Thus:

$$
\left|\chi_{0}\left(\tau, f_{d}\right)\right|=\left|\int_{-\infty}^{+\infty} S_{\alpha}(\varepsilon) S_{\alpha}^{*}(\varepsilon-u) e^{-j \varepsilon v} d \varepsilon\right|=\left|\chi_{\alpha}(u, v)\right|
$$

From formula (15), ambiguity function of the waveforms in the rotating waveform library can be calculated on ambiguity function of the traditional waveform.

We assumed that target location in delay-doppler plane is $\left[u_{0}, v_{0}\right]^{T}$, which is correspond to the maximum of $\left|\chi_{\alpha}(u, v)\right|$. Then the Fisher matrix is:

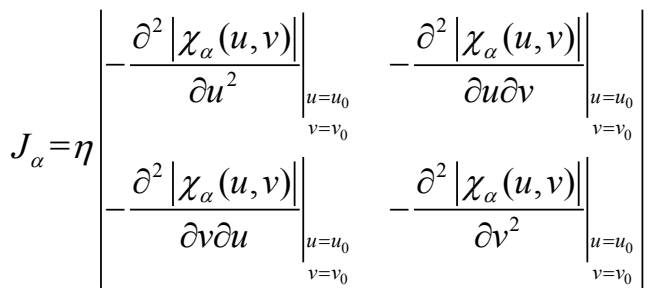

Where, $\eta$ is signal-noise ratio. Reference 16 indicated that the CRLB is the inverse of the Fisher matrix. Thus, the covariance matrix of the observation noise can be written $\mathrm{as}^{4}$ :

$$
R_{\alpha}\left(\psi_{k}\right)=T J_{\alpha}^{-1} T^{T}
$$

In (17), $\psi_{k}$ represents received signal at $k$ moment and $T=\left[\begin{array}{cc}\frac{c}{2} & 0 \\ 0 & \frac{c}{2 f_{c}}\end{array}\right]$ is transformation matrix, $c$ and $f_{c}$ represent the electromagnetic speed and carrier frequency respectively.

According the above analysis, the relation between waveforms in rotating waveform library and the traditional waveform is shown in Fig.1.Fig.1 indicates that CRLBs of the motion parameters estimation of the target for different waveforms in the rotating waveform library can be computed easily based on the traditional waveform. And according to the reference 4, CRLBs can be set the covariance matrix of the observation noise and it is likely to get the optimal solution of waveform parameters for waveform secluding. 


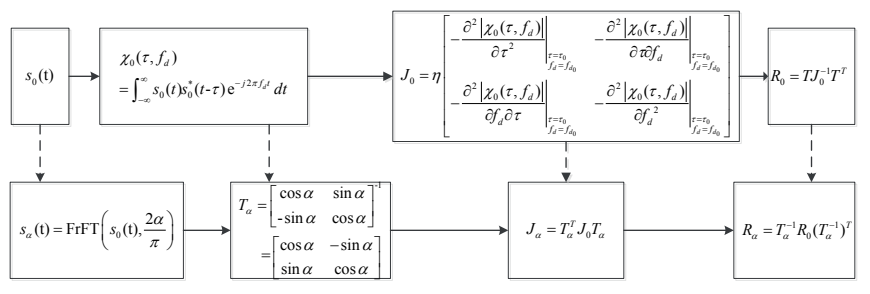

Fig.1. Relation between waveforms in the rotating waveform library and traditional

\section{PERFORMANCES ANALYSES OF WAVEFORM}

Ambiguity function is a kind of math tool for analyzing waveform performance effectively $[17,18]$. In this section, the ambiguity functions of waveforms in the rotating waveform libraries on polyphase codes (e.g. Barker 13 and Frank 16) are computed using mathematical derivation in section 3. And the performance of waveforms in the rotating waveform libraries is analyzed. The corresponding parameters are defined in table 1.

Tab 1. Definition of performance parameters

\begin{tabular}{|c|c|}
\hline Delay resolution & $A_{\tau}=\frac{\int_{-\infty}^{\infty}|\chi(\tau, 0)|^{2} d \tau}{|\chi(0,0)|^{2}}$ \\
\hline Doppler resolution & $\frac{\int_{-\infty}^{\infty}\left|\left(0, f_{d}\right)\right| d f_{d}}{|(0,0)|}$ \\
\hline Delay side lobe level & the level of the first side lobe in \\
\hline Doppler side lobe level & the level of the first side lobe in \\
& $|\chi(\tau, 0)|$
\end{tabular}

The corresponding parameters of Ambiguity function calculation are shown in table 2. In table 2, The amount of samples per bit is the parameter $r$. Other parameters are $F \times M \times t_{b}$, which represent the product between the maximum frequency shift, the sequence length and the bit duration, the total sequence duration is $\mathrm{T}$, as well as $\mathrm{N}$ and $\mathrm{K}$ are the total amount of delay and Doppler shifts respectively. The phase shifts of Barker 13 and Frank 16 are $[0,0,0,0,0,1,1,0,0,1,0,1,0]$ and $\left[0,0,0,0,0, \frac{\pi}{2}, \pi,-\frac{\pi}{2}, 0, \pi, 0, \pi, 0,-\frac{\pi}{2}, \pi, \frac{\pi}{2}\right.$ respectively. Performance parameters of waveforms in rotating waveform library based on Barker 13 and Frank 16 such as delay resolution, Doppler resolution are shown in Fig. 2 and Fig. 3 respectively. And $\mathrm{x}$-coordinate and $\mathrm{y}$-coordinate represent fractional order $p$ and parameter ratio $=\frac{\text { parameters of waveforms in waveform library }}{\text { parameters of traditional waveform }}$ respectively.

Tab 2. Parameters of waveform analysis

\begin{tabular}{|c|c|c|}
\hline parameters & Barker $\mathbf{1 3}$ & Frank 16 \\
\hline $\mathbf{r}$ & $50,100,200$ & $50,100,200$ \\
\hline $\mathbf{F} \times \mathbf{M} \times \mathbf{t}_{\mathbf{b}}$ & 20.02 & 24.96 \\
\hline $\mathbf{T}$ & 1 & 1 \\
\hline $\mathbf{N}$ & 130 & 160 \\
\hline $\mathbf{K}$ & 50 & 50 \\
\hline
\end{tabular}

Fig. 2 shows the performance of waveforms in rotating waveform library on Barker 13. Compared with canonical signal ( $p=0)$, many factors affect the performance of the waveforms. Subfigure (a1) shows that the delay resolution will improve with increasing the order $\mathrm{p}$. Moreover, the sampling rate $\mathrm{r}$ also affects the delay resolution. In the interval $0 \leq p \leq 0.7$, the delay resolution performance is improved significantly with increasing the sampling rate. Subfigure (a2) shows that in the interval $0.2 \leq p \leq 0.6$, the Doppler resolution can be slightly improved with increasing the order p. However, compared to the canonical waveform $(p=0)$, for Delay side lobe level and other important parametersthe fractional modulation causes lower performance in subfigure (a3). What's more, changing sample rate $r$ make no difference for Doppler SLL in (a4).

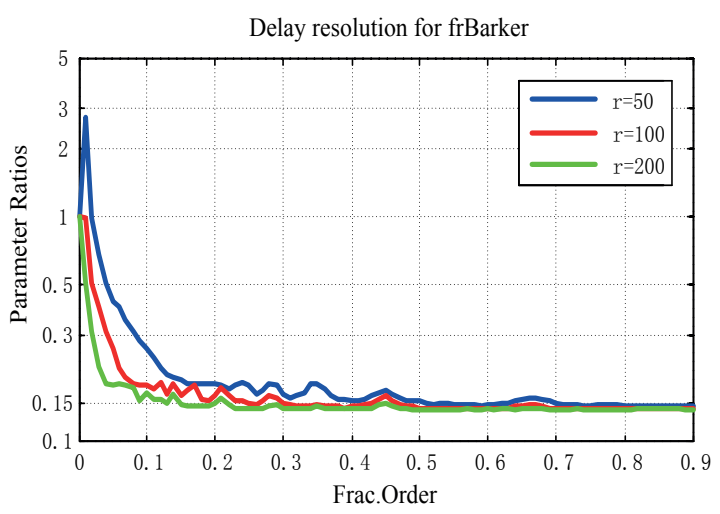

(a2)

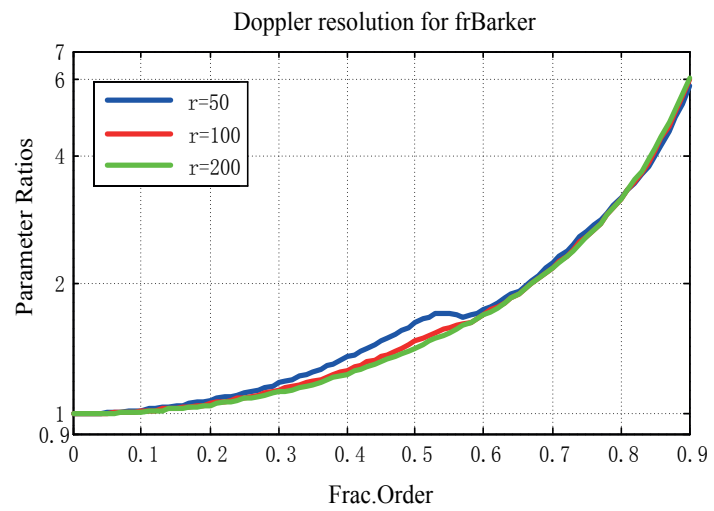

(a3)

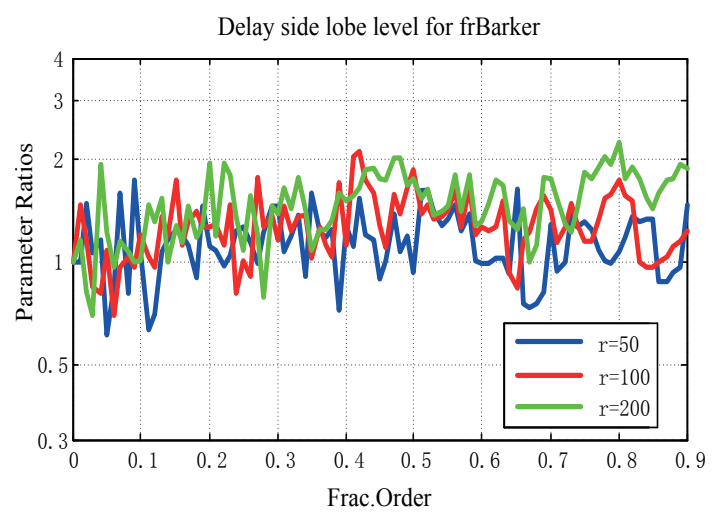


(a4)

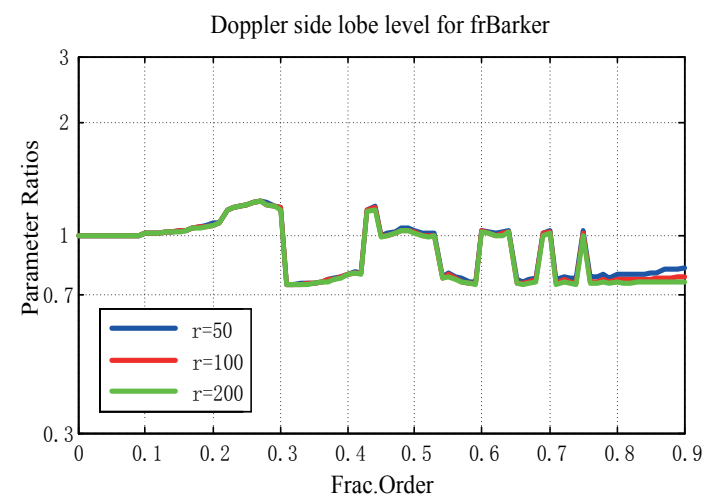

Fig.2. Performances ratios for frBarker (a1: Delay resolution; a2: Doppler resolution; a3: Delay side lobe level; a4: Doppler side lobe level)

Fig. 3 shows the performance of waveforms in rotating waveform library on Frank 16. Compared with canonical signal ( $p=0)$, many factors affect the performance of the waveforms. Subfigure (a1) shows that the delay resolution will improve with increasing the order $\mathrm{p}$. Moreover, the sampling rate $r$ also affects the delay resolution. In the interval $0 \leq p \leq 0.8$, the delay resolution performance is improved significantly with increasing the sampling rate. Subfigure (a2) shows that in the interval $0.1 \leq p \leq 0.6$, the Doppler resolution can be slightly improved with increasing the order p. However for other important parameters, such as Delay side lobe level, the fractional modulation results in lower performance compared to the canonical waveform $(p=0)$ in subfigure (a3). What's more, changing sample rate $r$ make no difference for Doppler SLL in (a4).

(a1)

Delay resolution for frFrank

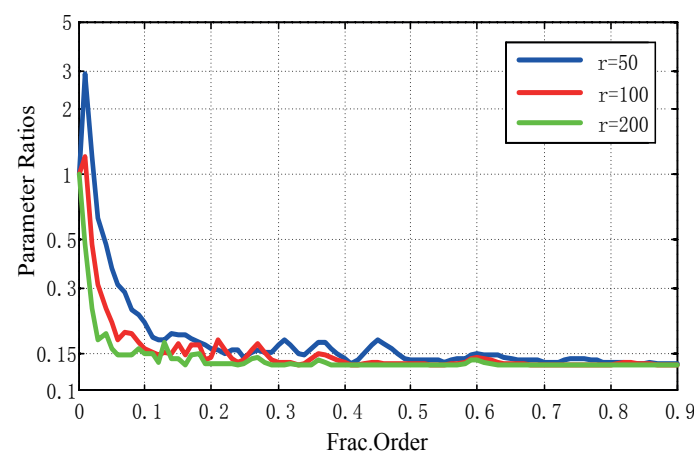

(a2)

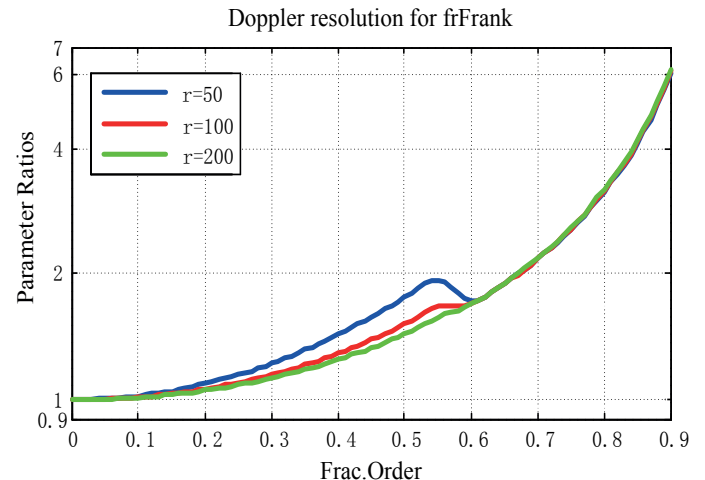

(a3)

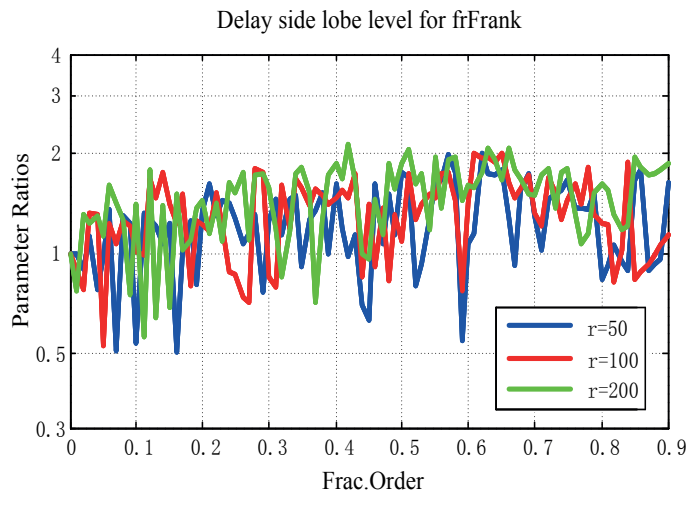

(a4)

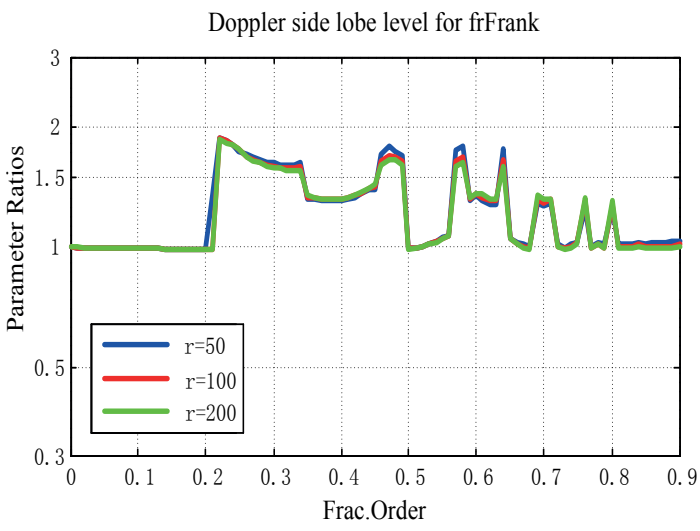

Fig.3. Performances ratios for frFrank (a1: Delay resolution; a2: Doppler resolution; a3: Delay side lobe level; a4: Doppler side lobe level)

Through the above analysis, compared to the canonical waveform $(\mathrm{p}=0)$, the delay resolution of waveform in rotating waveform library is improved significantly.

\section{ORTHOGONALITY ANALYSES OF WAVEFORMS}

This section is mainly to verify orthogonality between the waveforms in rotating waveform library. We assume that any two waveforms with different order $\mathrm{p}$ in rotating waveform library are orthogonal when their Peak Side Lobe Ratio (PSLR) of cross-correlation is lower than the PSLR of canonical waveform. The PSLR [19] is defined as:

$$
P S L R=10 \lg \left\{\frac{\max \left[R_{s}^{2}(m)\right]}{R_{0}^{2}(0)}\right\}
$$

In (18), $R_{s}$ represents the signal cross-correlation. SLLs of Barker 13 and Frank 16 used as thresholds in the analysis are shown in table 3:

Tab 3. SLLs of Barker 13 and Frank 16

\begin{tabular}{|c|c|}
\hline Sequence & SLL(dBs) \\
\hline Barker 13 & -22.28 \\
\hline Frank 16 & -21.07 \\
\hline
\end{tabular}


From Fig.4 and Fig.5,the number of orthogonal waveforms in the rotating waveform library on Frank 16 is more than the number of orthogonal waveforms of in the rotating waveform library on Barker 13.That is at same fractional order $\mathrm{p}$, orthogonality of the waveforms in the rotating waveform library on Frank 16 is better than that on Barker 13. And the orthogonality will be better with increasing the sampling rate $\mathrm{r}$.

The higher values of $r$ the number of chirped components in (4) subjoins, the mismatching between waveforms of the same libraries modulated with different fractional orders, resulting in a higher reuse factor, becomes stronger,leading to the influence above. In modern radar systems, a digital arbitrary waveform generator (AWG) can apply this framework. By using high speed D/A converters, the influences of the higher samples per chip rate $r$ can be addressed.

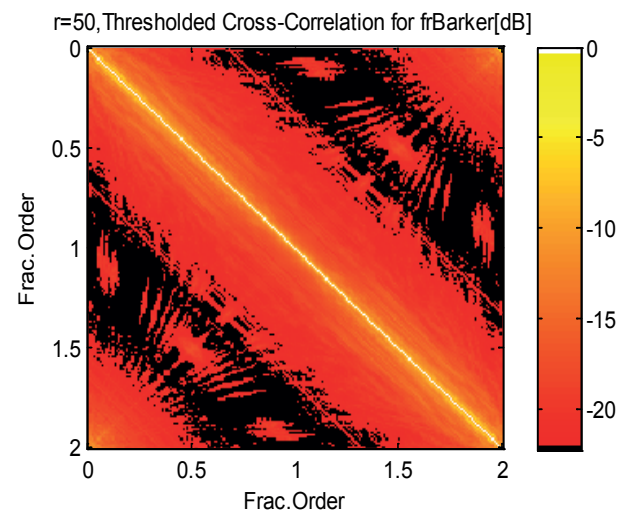

(a2)

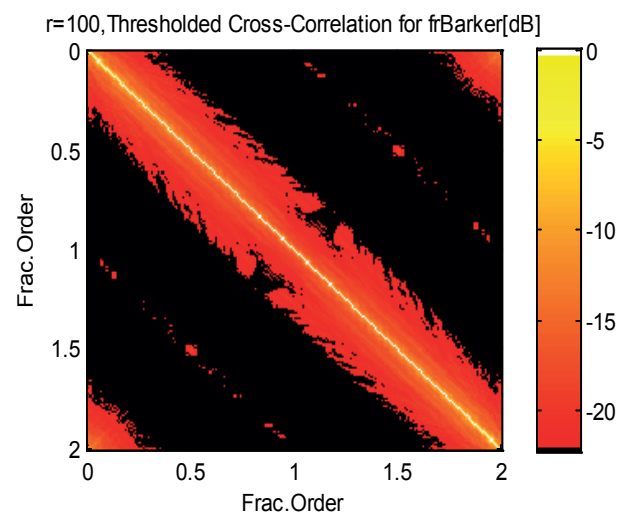

(a3)

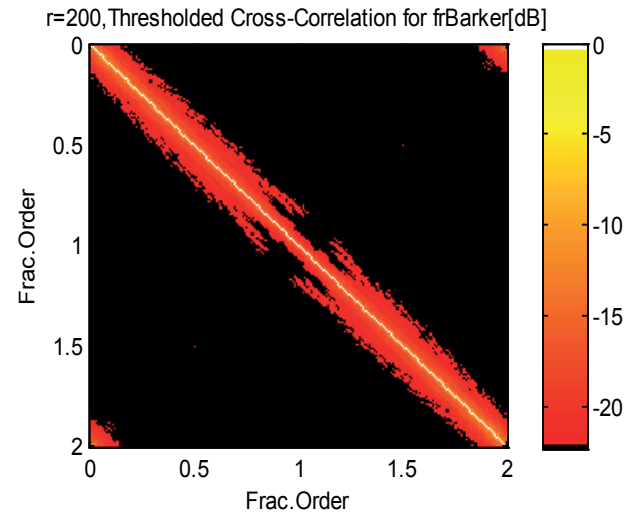

Fig.4. Orthogonality for frBarker ( $a 1: r=50 ; a 2: r=100 ; a 3: r 3=200$ ) (a1)

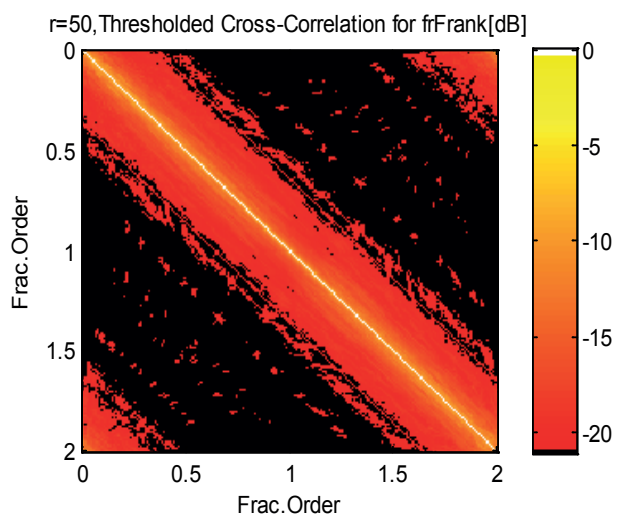

(a2)

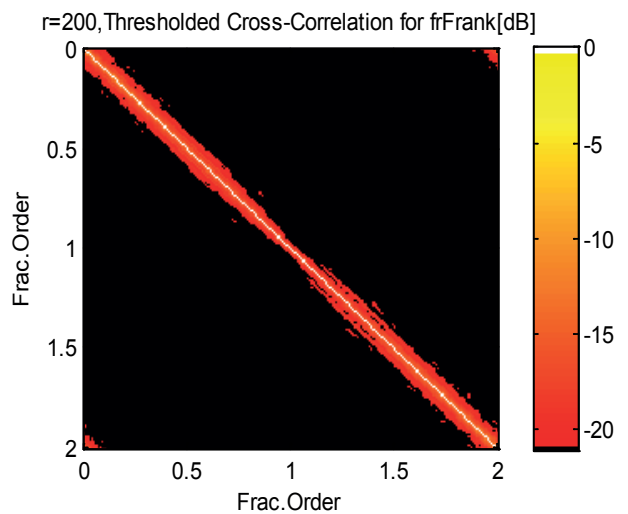

(a3)

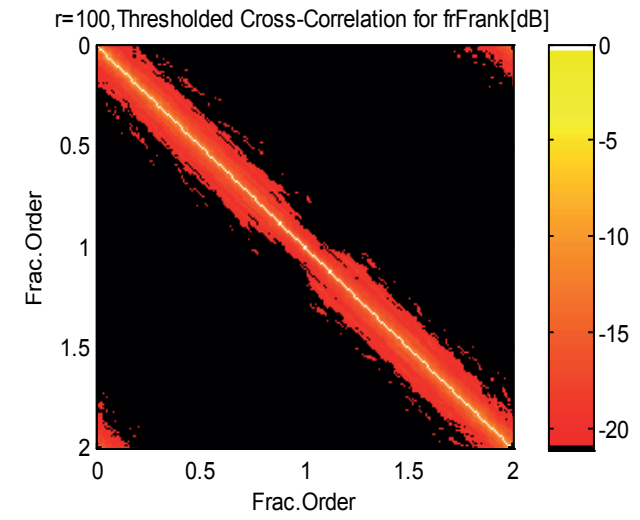

Fig.5. Orthogonality for frFrank (a1: $r=50$; $a 2: r=100 ; a 3: r 3=200$ )

Reuse interval is defined as the scope that make PSLR of waveforms at different order $p$ less than SLL of canonical signal.Fig. 6 and Fig.7 show the reuse interval of the rotating waveform library on Barker 13 and Frank 16 respectively. Whether the rotating waveform library on Barker 13 or Frank 16 , the reuse interval will increase with the increase of the sampling rate. It means that the waveform library $S$ is consist of more orthogonal waveforms with increasing $r$. Maximal reuse intervals of canonical signals for Barker 13 and Frank 16 are shown in table 4 . Table 4 shows that orthogonality of the rotating waveform library on Frank 16is better than that of the rotating waveform library on Barker 13. In some cases, such as reuse interval of 0.8 , table 4 indicates that () for a given code sequence, .more than 20 orthogonal waveforms can obtained 
Tab 4. Reuse interval of canonical waveform for each sequence

\begin{tabular}{|c|c|c|c|}
\hline Sequence & $\mathbf{r}=\mathbf{5 0}$ & $\mathbf{r}=\mathbf{1 0 0}$ & $\mathbf{r}=\mathbf{2 0 0}$ \\
\hline Barker 13 & 0.29 & 0.7 & 0.85 \\
\hline Frank 16 & 0.65 & 0.79 & 0.89 \\
\hline
\end{tabular}

(a1)

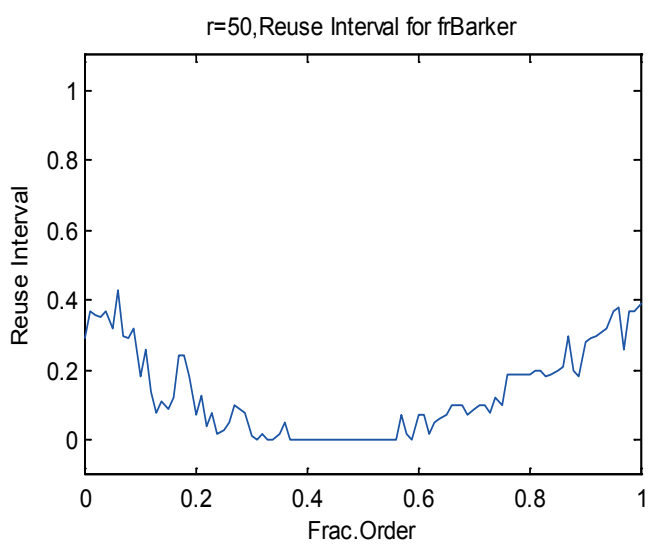

(a2)

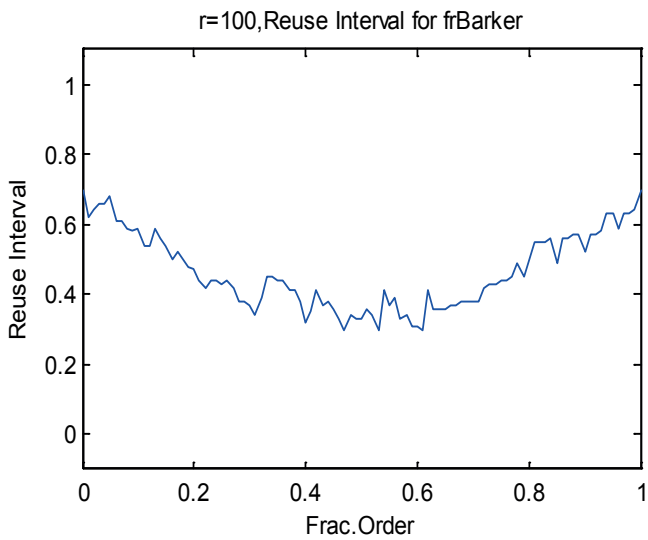

(a3)

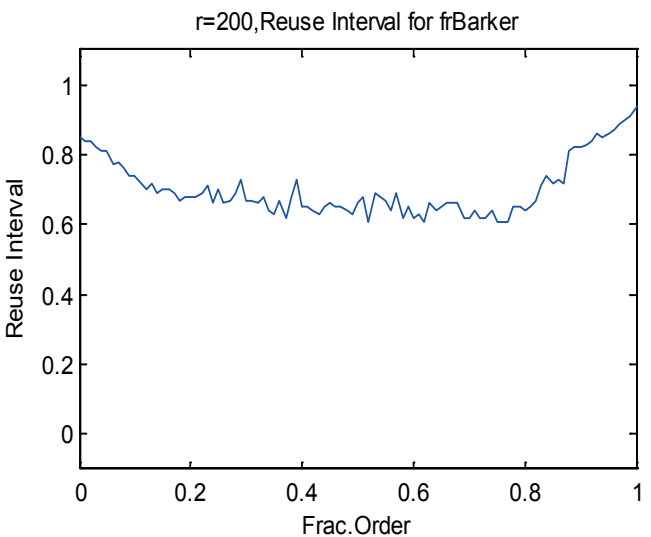

Fig.6. Reuse interval for frBarker (a1: $r=50 ; a 2: r=100 ; a 3: r 3=200$ ) (a1)

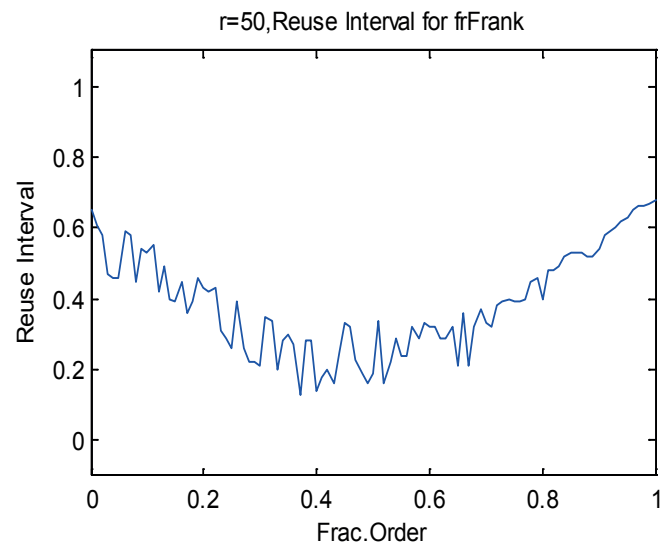

(a2)

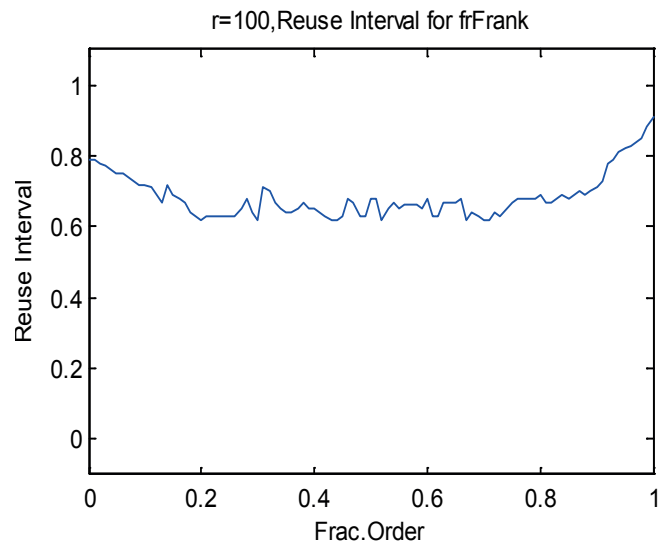

(a3)

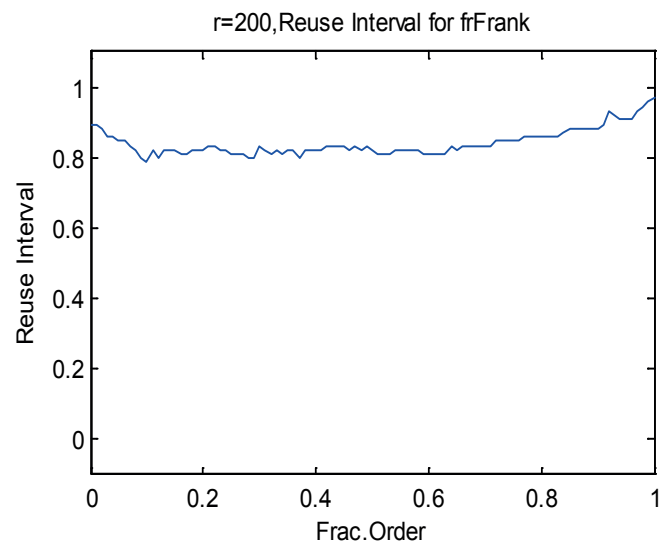

Fig.7. Reuse interval for frFrank (a1: $r=50 ; a 2: r=100 ; a 3: r 3=200$ )

\section{CONCLUSIONS}

In this paper based on the thoughts of agile radar waveform, we use FrFT to generate a rotating waveform libraries on Barker 13 or Frank 16. Then, the ambiguity function is used to analyze the performance of the waveforms in rotating waveform library such as the delay resolution. The orthogonality of the waveforms in rotating waveform library is analyzed too. The results show that the rotating waveform library introduces significant benefits fordelay 
resolution, orthogonality and reuse interval to meet the needs of different scenarios. Potential applications of the rotating waveform library are in the field of frequency reuse, agile tracking-Doppler systems, low probability of intercept radar and MIMO radar systems. For instance an arbitrary waveform provided by the novel libraries can be used by a LPI Radar. Moreover, because radar lacks of knowledge from the interceptor of fractional order used by the transmitter to increase its covertness,

\section{ACKNOWLEDGEMENTS}

This work is supported by Space Target Measurement Key Laboratory Foundation of China.

\section{BIBLIOGRAPHY}

1. C.H. Chen, Q. Zhang, Y. Luo.: A waveform optimization designing method for cognitive radar with steppedfrequency signal. Acta Aeronautica et Astronautica Sinica, No 7, Vol 37, 2016, p. 2276-2285

2. S. Haykin.: Cognitive radar: a way of the future. IEEE Signal Processing Magazine., No 1, Vol 23, 2006, p. 30-40

3. J. Shang, D. Zhao, Y. Wei.: Pareto-Optimal Sparse Frequency Radar Waveform Design. Systems Engineering and Electronics, No 7, Vol 38, 2016, p. 1538-1542

4. D. Cochran, S. Suvorova, SD. Howard, B. Moran.: Waveform Libraries. IEEE Signal Processing Magazine, No 1, Vol 26, 2009, p. 12-21

5. X. Li, M.M. Fan.: Research Advance on Cognitive Radar and Its Key Technology. ACTA ELECTRONICA SINICA, No 9, Vol 40, 2012, p. 1863-1870

6. H.E. Xia.: Adaptive Waveform Selection Techniques for Target Tracking. ChangSha, 2010.

7. D.B. Yu, Y.H. Wu, W.G. Zhu: Research on Selection Methods of Target Tracking Waveforms Based on a Waveform Library. RADAR \& ECM, No 2, Vol 33, 2013, p. $35-42$

8. A.A.A. Solyman, S. Weiss, J.J. Soraghan.: Low-Complexity LSMR Equalisation of FrFT-Based Multicarrier Systems in Doubly Dispersive Channels. IEEE International Symposium on Signal Processing and Information Technology, ISSPIT 2011, p. 461-465, 2011

9. B. Jin.: Research on Target Tracking Methods in Cognitive Radar [D]. Xian, Xian Electronics Science and Technology University, 2014.

10. C.V. Ilioudis, C. Clemente, I. Proudler, J.J. Soraghan.: Performance Analysis of Fractional Waveform Libraries in MIMO Radar Scenario. IEEE National Radar Conference - Proceedings, v 2015-June, June, p. 1119-1124

11. C.V. Ilioudis, C. Clemente, I. Proudler, J.J. Soraghan.: Radar Waveform Libraries Using Fractional Fourier Transform. in 2014 IEEE Radar Conference, Cincinnati, Ohio, 19-23 May 2014.

12. F. Zhang, R. Tao, Y. Wang.: Angle Resolution of Fractional Fourier Transform. 2014 31th URSI General Assembly and Scientific Symposium, URSI GASS 2014, October 17, 2014

13. Zhang. Lili, Liu Sixin, Qu, Lete, et al.: Research on wavelet extraction of gpr signals based on multilevel fractional fourier transform filter [J]. Journal of the Balkan Tribological Assocaitaion, Vol. 22, No 1, 2016, p. 807-818

14. H.M. Ozaktas, M.A. Kutay, D. Mendlovic.: Introduction to the Fractional Fourier Transform and its Applications. IEEE Signal Processing Magazine., No 9, Vol 44, 1996, p. 2141-2150.

15. L.B. Almeida.: The fractional Fourier Transform and TimeFrequency Representations. IEEE Transactions on Signal Processing., No 11, Vol 42, 1994, p. 3084-3091

16. H.L.V. Trees.: Detection, Estimation, and Modulation Theory, Part III. New York, 2001

17. X.Y. Li, Y.L. Dong, L. Zhang, J. Guan.: A New Design Method of Low Sidelobe Level LFM Noise Radar Waveform. Journal of Electronics \& Information Technology, No 6, Vol 38, 2016, p. 1452-1459

18. C. Gao, K.C. The, A. Liu, H. Sun.: Piecewise LFM Waveform for MIMO Radar. IEEE Transactions on Aerospace and Electronic Systems., No 2, Vol 52, 2016, p. 590-602

19. J. Yang, Z. Qiu, X. LI, Z. Zhuang.: Analysis and Processing of the Chaotic-Based Random Stepped Frequency Signal. Journal of National University of Defense Technology, No 6, Vol 34, 2012, p. 163-169

\section{CONTACT WITH AUTHOR}

\section{Wu yumin}

e-mail:wuyumin@stu.xmu.edu.cn Communication Engineering Xiamen University

Xiamen Fujian 361005 China 